\title{
Seeing Double: A Rare Presentation of Non-Hodgkin Lymphoma
}

Fitzgerald LA ${ }^{1 *}$ and Stephens $\mathrm{DM}^{2}$

${ }^{1}$ Department of Internal Medicine, University of Utah, USA

${ }^{2}$ Division of Hematology and Hematologic Malignancies, Huntsman Cancer Institute, USA

*Corresponding author: Fitzgerald LA, Department of Internal Medicine, University of Utah, 30 North 1900 East, Room 4C104, Salt Lake City, USA

Received: May 13, 2017; Accepted: J une 12, 2017; Published: J une 22, 2017

\begin{abstract}
Background: This is the case of a new diagnosis of aggressive nonHodgkin lymphoma presenting as acute neurologic deficits. Upon evaluation, the patient is found to be suffering from Neurolymphomatosis, a rare manifestation of lymphoma characterized by direct invasion of the nerve roots by lymphoma cells. This case is unique in that it is even rarer to have neurolymphomatosis as the presentation of new lymphoma diagnosis.
\end{abstract}

Case Presentation: A 65 year-old female presents with acute-onset diplopia and slurred speech. Upon evaluation for stroke, an incidental chest mass is found. Biopsy of the mass is consistent with Burkitt Lymphoma and her neurologic deficits are diagnosed as Neurolymphomatosis. Unfortunately, without rapid and aggressive therapy, the patient succumbed to her disease.

Conclusion: Neurolymphomatosis is a rare manifestation of non-Hodgkin lymphomas and an even rarer presentation of a new lymphoma diagnosis. It should remain on the differential diagnosis of secondary central nervous system involvement of lymphoma as the rapid recognition of this disease process is critical for mitigating morbidity and mortality.

Keywords: Neurolymphomatosis; Non-Hodgkin lymphoma; Burkitt lymphoma; CNS involvement by lymphoma

\section{Abbreviations}

CN: Cranial Nerve; CT: Computed Tomography; NHL: NonHodgkin's Lymphoma; CNS: Central Nervous System; R-CHOP: Rituximab, Cyclophosphamide, Doxorubicin, Vincristine, and Prednisone; CODOX-M: Cyclophosphamide, Vincristine, Doxorubicin, High-dose Methotrexate; IVAC: Ifosfamide, Cytarabine, Etoposide, and Intrathecal Methotrexate

\section{Case Presentation}

A 65 year-old female with a history of liver cirrhosis presented with acute-onset double vision and slurred speech. The stroke response team was activated in the Emergency Department. Despite initial concerns, however, her clinical picture would become less consistent with acute stroke. The patient's past medical history is notable for cirrhosis complicated by ascites, esophageal varices, and chronic thrombocytopenia. Her cirrhosis was secondary to Hepatitis $\mathrm{C}$ infection, which had been treated prior to presentation. She had a 40 pack-year smoking history and reported quitting one month prior to presentation. In addition to the above, the patient also endorsed headache, abdominal pain, and nausea upon presentation. Review of systems was negative for fever, confusion, shortness of breath, cough, hemoptysis, or chest pain.

Upon presentation, the patient was afebrile with vital signs notable only for mild hypertension. She was alert and oriented to person, place, and time and was noted to be in mild distress. Remarkable findings on examination included inward gaze of the right eye [cranial nerve $(\mathrm{CN}) \mathrm{VI}$ palsy], mild right-sided facial droop (CN VII palsy), and mild slurring of speech (CN IX/X). Strength was full and symmetric. Sensation was intact. She was given a $\mathrm{NIH}$ Stroke Scale score of 5. Laboratory analysis was significant for a normal WBC and a platelet count of 99,000 cells $/ \mathrm{mm}^{3}$, which was at baseline. Radiographic studies included unremarkable CT scan of the head, CT angiogram of the head/neck with no findings suggestive of acute ischemia, and chest X-Ray with right-sided opacity and pleural effusion. Incidentally, the CT angiogram revealed apartially visualized large mediastinal mass (Figure 1).

The patient was admitted for neurologic deficits of unclear etiology. Brain MRI showed no findings of ischemia, hemorrhage, or parenchymal process and was only notable for mild white matter disease felt to represent chronic microvascular ischemic change. A lumbar puncture was performed and fluid analysis showed normal protein, glucose, and cell cytology. Flow cytometry was not performed

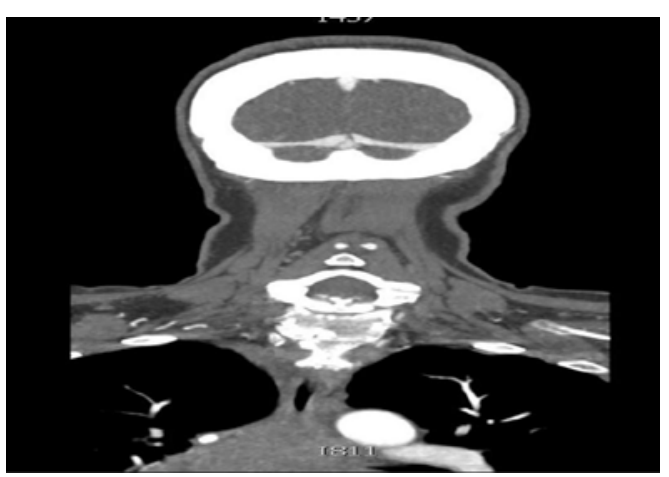

Figure 1: CT Angiogram of the Head and Neck reveals superior portion of a large mediastinal mass. 


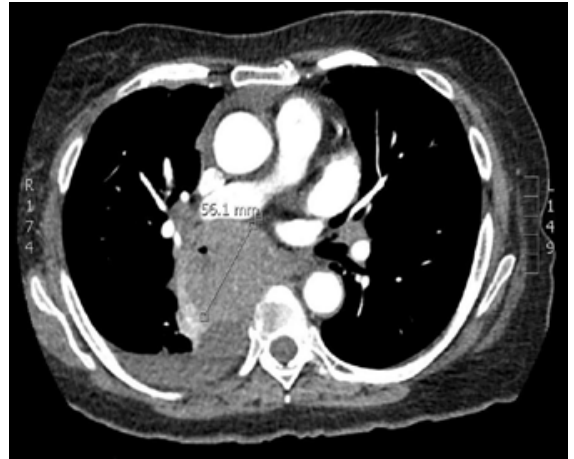

Figure 2: CT of the Chest further characterizes the large mediastinal mass Right pleural effusion is also noted.



Figure 3: Sagittal view of PET-CT showing extensive disease burden including lesion at skull base.

on this sample. To further evaluate the incidentally-found mediastinal mass, a CT chest was performed. It showed a $5.6 \mathrm{~cm}$ subcarinal mass (Figure 2).This finding raised the possibility of malignancy. As the likelihood of a primary central nervous system (CNS) process (including stroke, mass, infectious process) causing the patient's neurologic deficits waned, our differential diagnosis shifted to include processes connected to this newly found chest lesion.

The first step in narrowing our neurologic differential diagnosis was determining the etiology of the chest mass. Given the patient's smoking history, small cell lung cancer was considered. Thymoma with a presentation akin to myasthenia gravis also fit. Lymphoma was also on the differential given the location of the mass. A transbronchial fine needle aspiration was performed. Initial flow cytometry showed a monoclonal $\mathrm{CD} 10+\mathrm{B}$-cell population suggestive of a high-grade B-cell non-Hodgkin's lymphoma (NHL). Unfortunately, the sample was inadequate to further delineate the NHL sub-classification.

Meanwhile, the patient's clinical status continued to deteriorate as she developed additional cranial nerve palsies, which were thought to be related to her newly diagnosed NHL. A PET-CT was obtained and showed wide-spread disease including two symmetric lesions at the base of the skull (Figure 3). A biopsy of an involved lymph node was performed in order to sub-classify her NHL. It became clear that emergent treatment of the patient's underlying malignancy would be essential to slow her clinical decline and treat her neurologic deficits.

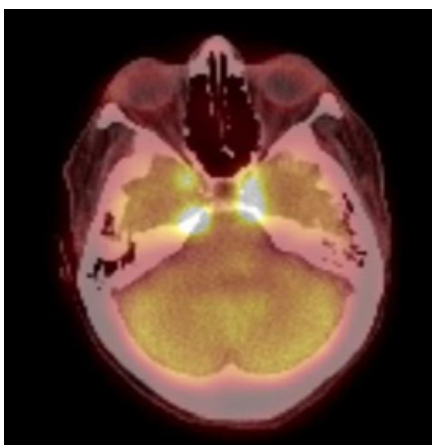

Figure 4: Axial PET-CT view of brain showing increased signal intensity near the bilateral superior orbital fissures.

While expeditiously working up her NHL diagnosis, we considered our differential diagnosis. Secondary involvement of the nervous system by NHL can manifest in multiple ways, including: leptomeningeal metastasis, parenchymal brain metastasis, intramedullary spine metastasis, neurolymphomatosis, and paraneoplastic disease (Table 1) [1]. Whereas leptomeningeal metastasis is the most common CNS manifestation of NHL and often presents with cranial nerve palsies, it typically will exhibit nerve enhancement on MRI and abnormal cerebrospinal fluid studies, including malignant cells on cytology. The patient's studies were not supportive of this manifestation. Her MRI also did not show contrast enhancing lesions as one would expect with parenchymal brain metastasis. Additionally, intramedullary spinal metastases would not present with cranial nerve palsies, given anatomic location.

A paraneoplastic process was high on our differential diagnosis. The patient's blood was tested for voltage-gated calcium channel antibodies to assay for Lambert-Eaton myasthenic syndrome and acetylcholine receptor antibodies for myasthenia gravis. Although not a paraneoplastic process, neurosarcoidosis was also considered and a serum ACE level was ordered. These studies all returned normal. Although absence of these antibodies does not exclude a paraneoplastic process, the lack of encephalitis-related changes on brain MRI and inflammatory changes in CSF made it less likely.

As other diagnoses were systematically eliminated, neurolymphomatosis became our leading diagnosis for the patient's neurologic deficits in the context of lymphoma. Neurolymphomatosis is a rare manifestation of NHL and is defined as the direct invasion of peripheral nerve roots by malignant lymphoma cells, typically involving the cranial or spinal nerve roots [2]. The most common presentations include: peripheral neuropathy or radiculopathy, cranial neuropathy, or painless polyneuropathy [3]. In a large retrospective study on neurolymphomatosis, the majority of cases were due to B-cell NHL. Neurolymphomatosis was the initial presenting feature in $26 \%$ of those cases [3]. The diagnosis of neurolymphomatosis is difficult and requires a combination of a high degree of clinical suspicion, multimodal imaging including PET-CT, and, if feasible, a nerve biopsy [2]. Typically, conventional MRI and CT imaging are normal. PET-CT may show enhancement of involved nerves, although may also lack specific findings.

In our patient, PET-CT showed enhancement at the skull base where cranial nerves III, IV, and VI enter the superior orbital 
Table 1: The differential diagnosis of secondary involvement of the central nervous system by Non-Hodgkin lymphoma.

\begin{tabular}{|c|c|c|c|c|}
\hline Process & Prevalence in NHL & Presentation & Imaging Findings & CSF Findings \\
\hline Leptomeningeal Metastasis & $8 \%[1]$ & CN Palsy & Nerve Enhancement & $\begin{array}{c}\uparrow \text { opening pressure, } \downarrow \text { glucose, } \uparrow \text { protein, } \\
\text { lymphocytic pleocytosis, malignant cells } \\
\text { on cytology }\end{array}$ \\
\hline Brain Metastasis & $1.4-43 \%[1]$ & $\begin{array}{l}\text { Seizures; focal sensory, } \\
\text { motor, or CN deficits }\end{array}$ & $\begin{array}{c}\text { Contrast-enhancing lesions on MRI } \\
\text { Brain }\end{array}$ & Typically unremarkable \\
\hline $\begin{array}{l}\text { Intramedullary Spinal } \\
\text { Metastasis }\end{array}$ & Rare & $\begin{array}{l}\text { Pain, weakness, } \\
\text { spasticity, bowel/bladder } \\
\text { symptoms }\end{array}$ & $\begin{array}{l}\text { Contrast-enhancing lesions on MRI } \\
\text { Spine }\end{array}$ & Typically unremarkable \\
\hline Neurolymphomatosis & Rare & Painful Radiculopathy & $\begin{array}{c}\text { Normal or may have nerve } \\
\text { thickening or increased signaling } \\
\text { on PET-CT }\end{array}$ & Normal \\
\hline Paraneoplastic Process & Rare & Dependent on Process & $\begin{array}{c}\text { Normal or increased signal on } \\
\text { FLAIR MRI }\end{array}$ & $\begin{array}{c}\text { May see } \uparrow \text { protein, pleocytosis; +/- } \\
\text { antibodies [4] }\end{array}$ \\
\hline
\end{tabular}

fissure (Figure 4). Her final diagnosis and source of diplopia was neurolymphomatosis - the direct invasion of the cranial nerve roots.

\section{Conclusion}

Management of neurolymphomatosis relies on prompt recognition, as it is imperative to provide rapid treatment of the underlying lymphoma to prevent further neurologic deterioration and possibly prolong survival. While awaiting the results of the pelvic lymph node biopsy, the patient was given one cycle of R-CHOP (Rituximab, Cyclophosphamide, Doxorubicin, Vincristine, and Prednisone) for treatment of high-grade B-cell lymphoma. Her poor performance status at the time precluded more intensive regimens. She also received palliative radiation to her skull base for symptom management. Her neurologic deficits slightly improved with radiotherapy. Eventually, her biopsy returned consistent with the diagnosis of Burkitt Lymphoma, a highly aggressive B-cell lymphoma that is often unresponsive to R-CHOP [5].Unfortunately, the patient did not tolerate an aggressive chemotherapy regimen and declined further treatment. She died less than three months after initial presentation.

The ideal treatment for this patient would have been CODOX-M (cyclophosphamide, vincristine, doxorubicin, highdose methotrexate) with IVAC (ifosfamide, cytarabine, etoposide, and intrathecal methotrexate), also known as the Magrath Regimen. This regimen not only has high cure rates for Burkitt Lymphoma, but includes several CNS-penetrating drugs (methotrexate, cytarabine, ifosfamide, and etoposide) that may have more effectively targeted the patient's CNS disease [6]. Unfortunately, the significant toxicities associated with this regimen prohibited its use in this patient secondary to her poor performance status and medical comorbidity.
This case demonstrates a rare presentation of NHL in the form of neurolymphomatosis. Despite absence of findings on conventional imaging and CSF studies, a high suspicion for malignant involvement of the CNS remained. This case underscores the significance of prompt recognition of neurolymphomatosis so that treatment may be initiated rapidly to prevent further neurologic morbidity. Although this patient presented with advanced disease, it remains unknown if expeditiously reaching a diagnosis of Burkitt Lymphoma would have led to earlier, disease-specific treatment and thus lessened her neurologic morbidities and prevented her mortality.

\section{References}

1. Hochberg E, Hochberg F. Clinical presentation and diagnosis of secondary central nervous system lymphoma. In: Post TW (edn), Waltham MA. 2017

2. Shree R, Goyal MK, Modi M, Gaspar BL, Radotra BD, Ahuja CK, et al. The Diagnostic Dilemma of Neurolymphomatosis. J Clin Neurol. 2016; 12: 274 281.

3. Grisariu S, Avni B, Bachelor TT, van den Bent MJ, Bokstein F, Schiff D, Kuittinen $\mathrm{O}$, et al. Neurolymphomatosis: an International Primary CNS Lymphoma Collaborative Group report. Blood. 2010; 115: 5005-5011.

4. McKeon A, Pittock SJ, Lennon VA. CSF complements serum for evaluating paraneoplastic antibodies and NMO-IgG. Neurology. 2011; 76: 1108

5. Bishop PC, Rao VK, Wilson WH. Burkitt's lymphoma: molecular pathogenesis and treatment. Cancer Invest 2000; 18; 574.

6. Mead GM, Sydes MR, Walewski J, Grigg A, Hatton CS, Pescosta N, et al. An international evaluation of CODOX-M and CODOX-M alternating with IVAC in adult Burkitt's lymphoma: results of United Kingdom Lymphoma Group LY06 study. Ann Oncol. 2002; 131264.
Ann Hematol Oncol - Volume 4 Issue 6 - 2017 ISSN : 2375-7965 | www.austinpublishing group.com Fitzgerald et al. (@ All rights are reserved Hematol Oncol. 2017; 4(6): 1155. 